\title{
Marginal Stability and Stabilization in the Numerical Integration of Ordinary Differential Equations
}

\author{
By H. Brunner*
}

\begin{abstract}
Strongly stable and consistent multistep methods with maximum order are subject to marginal (or weak) stability. In this paper we introduce modified multistep methods whose coefficients depend linearly on the stepsize $h$ and a parameter $L$ in such a way that the order of the original method is not decreased. By choosing $L$ in a suitable manner (depending essentially on $f_{y}(x, y)$ of the differential equation $y^{\prime}=f(x, y)$ and on the growth parameters of the multistep method), marginal stability can be eliminated.
\end{abstract}

1. Introduction. In [3], G. Dahlquist introduced a class of recurrence relations of the form

$$
\sum_{\nu=0}^{k} \alpha_{\nu} y_{n+\nu}=h \sum_{\nu=0}^{k} \beta_{\nu} f_{n+\nu}, \quad(n=0,1, \cdots),
$$

where the coefficients $\alpha_{v}, \beta_{v}(\nu=0,1, \cdots, k)$ are real and independent of $n, \alpha_{k} \neq 0$, $f_{m}=f\left(x_{m}, y_{m}\right), x_{m}=a+m h, h>0$. These relations, usually called linear $k$-step methods, are used to generate approximations to the exact solution of the initialvalue problem

$$
y^{\prime}=f(x, y), \quad y(a)=s, \quad a \leqq x \leqq b \quad\left({ }^{\prime}=d / d x\right)
$$

at the points $x=x_{m}, x_{m} \in[a, b]$.

We assume that the function $f(x, y)$ be defined and continuous in the strip $S$ : $a \leqq x \leqq b,|y|<\infty$. In $S$, it shall also satisfy a Lipschitz condition with respect to the variable $y$.

In the following we shall denote the exact solution of (1.2) at $x=x_{m}$ by $y\left(x_{m}\right)$, whereas $y_{m}$ means the approximation to $y\left(x_{m}\right)$ given by (1.1) and a certain set of initial values $y_{0}, \cdots, y_{k-1}$.

The characteristic polynomials associated with (1.1) are defined by

$$
\begin{aligned}
& \rho(z) \equiv \sum_{\nu=0}^{k} \alpha_{\nu} z^{\nu} \quad\left(\alpha_{k} \neq 0\right), \\
& \sigma(z) \equiv \sum_{\nu=0}^{k} \beta_{\nu} z^{\nu} .
\end{aligned}
$$

The $k$-step method (1.1) will be referred to as the method $(\rho, \sigma)$.

Definition. Let

$$
\mathfrak{L}[y(x) ; h] \equiv \sum_{\nu=0}^{k}\left(\alpha_{\nu} y(x+\nu h)-h \beta_{\nu} y^{\prime}(x+\nu h)\right) .
$$

Received December 1, 1969.

AMS Subject Classifications. Primary 6560, 6561.

Key Words and Phrases. Nonlinear ordinary differential equations of order one, numerical solution, optimal multistep methods, marginal stability, stabilization.

* This work was supported by the National Research Council of Canada (Grant A4805). 
Then the largest integer $p$, for which

$$
\operatorname{Max}_{x_{n} \in[a, b]}\left|\mathscr{L}\left[y\left(x_{n}\right) ; h\right]\right|=O\left(h^{p+1}\right)
$$

for all solutions of (1.2) having continuous derivatives of sufficiently high order, is called the order of the method $(\rho, \sigma)$.

Definition. The method $(\rho, \sigma)$ is called (strongly) stable if no zero of $\rho(z)$ has modulus exceeding one and if the zeros on the unit circle are simple.

The method $(\rho, \sigma)$ is called consistent if

$$
\rho(1)=0, \quad \rho^{\prime}(1)=\sigma(1) .
$$

It is known [3, p. 51] that the order of a stable and consistent $k$-step method cannot exceed $p=k+2$. A method $(\rho, \sigma)$ has the maximum order $p=k+2$ if its characteristic polynomials satisfy the following conditions:

(i) The degree $k$ of $\rho(z)$ is an even number $(k \geqq 2)$.

(ii) The zeros $z_{i}(j=1, \cdots, k)$ of $\rho(z)$ have all modulus one.

(iii) If

$$
\rho(z) \cdot(\log z)^{-1}=\sum_{\nu=0}^{\infty} c_{\nu} \cdot(z-1)^{\nu} \quad(\log 1=0),
$$

then

$$
\sigma(z)=\sum_{\nu=0}^{k} c_{\nu} \cdot(z-1)^{\nu} .
$$

Definition. A stable and consistent $k$-step method $(\rho, \sigma)$ with maximum order $p=k+2$ is called an optimal method.

Throughout this paper, the method $(\rho, \sigma)$ is assumed to be an optimal method.

It is well known that optimal $k$-step methods are subject to marginal (or weak) stability (see, for instance, [5, p. 242]). The main reason for this behavior is that some of the growth parameters

$$
\lambda_{i}=\frac{\sigma\left(z_{i}\right)}{z_{j} \cdot \rho^{\prime}\left(z_{i}\right)}, \quad j=1, \cdots, k \quad\left(\rho\left(z_{i}\right)=0\right),
$$

(which are known to be real, [4, p. 40]) are negative. In particular, the growth parameter associated with the zero $z=-1$ of $\rho(z)$ satisfies the inequality $\lambda \leqq-\frac{1}{3}$, where equality is reached only for $k=2$.

In [1], the author has derived a class of modified $k$-step methods. These methods are given by the characteristic polynomials

$$
R(w, h L) \equiv \sum_{\nu=0}^{k} \tilde{\alpha}_{\nu} w^{\nu}=\rho(w)+\frac{h L}{2} \cdot \rho^{*}(w)
$$

and

$$
S(w, h L) \equiv \sum_{v=0}^{k} \tilde{\beta}_{\nu} w^{\nu}=\sigma(w)+\frac{h L}{2} \cdot \sigma^{*}(w)
$$

Here

$$
\rho^{*}(w) \equiv \sum_{v=0}^{k} p_{v} \cdot w^{v}=(w-1) \cdot \rho^{\prime}(w)
$$


and

$$
\sigma^{*}(w) \equiv \sum_{\nu=1}^{k} q_{\nu} \cdot w^{\nu}=\sum_{\nu=0}^{k} \gamma_{\nu}(w-1)^{\nu},
$$

with

$$
\rho^{*}(w) \cdot(\log w)^{-1}=\sum_{\nu=0}^{\infty} \gamma_{\nu} \cdot(w-1)^{\nu} \quad(\log 1=0) .
$$

The nonnegative parameter $L$ is called the parameter of stabilization. Obviously the coefficients of the polynomials $R(w, h L)$ and $S(w, h L)$ are linear functions of $h L$, and we have $R(w, 0)=\rho(w), S(w, 0)=\sigma(w)$. It has been shown [1, p. 442] that the modified $k$-step method $(R, S)$ has order $p=k+2$, and for the case where $f(x, y)=$ $A y(A<0)$ the author has demonstrated that marginal stability can be eliminated (without decreasing the order of the $k$-step method) by choosing the parameter $L$ in a suitable manner depending essentially on the growth parameters of the original $k$-step method and on the constant $A$.

The purpose of this paper is to extend the results of [1] to nonlinear ordinary differential equations of order one. In the following section some important properties of the polynomials $R(w, h L)$ and $S(w, h L)$ are presented. In Section 3 the initial-value problem (1.2) is solved by the modified method $(R, S)$, and an asymptotic relation for the discretization error is found (asymptotic in the sense $h \rightarrow 0, n h$ fixed, $L$ fixed). From this result conditions for the parameter $L$ will be derived in order to eliminate marginal stability with respect to the given differential equation. The last section of the paper contains some numerical illustrations.

2. Properties of the Polynomials $R(w, h L)$ and $S(w, h L)$.

LEMMA 1. The $k$-step method $(R, S)$ defined by (1.7) and (1.8) is consistent, i.e.,

$$
R(1, h L)=0
$$

and

$$
R^{\prime}(1, h L)=S(1, h L) \quad\left({ }^{\prime}=d / d w\right) .
$$

The proof is obvious and therefore omitted.

LEMMA 2. There exists $a \Omega>0$ such that the zeros $w_{i}=w_{i}(h L)(j=1, \cdots, k)$ of $R(w, h L)$ have multiplicity one for all $h L \in[0, \Omega)$.

Proof. From (1.7) and (1.9) we have

and

$$
R(w, h L)=\sum_{\nu=0}^{k}\left(\alpha_{\nu}+\frac{h L}{2} \cdot p_{\nu}\right) \cdot w^{\nu}
$$

$$
\rho(w)=R(w, 0)=\sum_{\nu=0}^{k} \alpha_{\nu} \cdot w^{\nu}
$$

Since the zeros of $\rho(w)$ are simple by hypothesis, the result follows from the fact that the zeros of the polynomial $R(w, h L)$ are continuous functions of $h L$ (see also [7, p. 3]).

LEMmA 3. For $h L>0$ and $j=2, \cdots, k$, the zeros $w_{i}=w_{i}(h L)$ of $R(w, h L)$ are contained in the interior of the unit circle, whereas the zero $w_{1}=1$ is independent of $h L$. 
Proof. By (1.7), the zeros of $R(w, h L)$ are given by the zeros of

$$
\rho(w)+\frac{h L}{2}(w-1) \cdot \rho^{\prime}(w)=(w-1) \cdot \rho(w) \cdot\left[\frac{1}{w-1}+\frac{h L}{2} \cdot \sum_{j=1}^{k} \frac{1}{w-z_{j}}\right],
$$

where $\rho\left(z_{i}\right)=0$ and $\left|z_{j}\right|=1, j=1, \cdots, k$. Let

$$
F(w)=\frac{1}{w-1}+\frac{h L}{2} \cdot \sum_{j=1}^{k} \frac{1}{w-z_{j}} .
$$

For $h L>0$, it follows [7, p. 22] that each zero of $F(w)$ lies in the convex hull $H$ of the points $1, z_{1}, \cdots, z_{k}$. No zero lies on the boundary of $H$ except the zero $w_{1}=1$. This completes the proof.

Definition. The numbers

$$
\tilde{\lambda}_{i} \equiv \frac{S\left(w_{i}, h L\right)}{w_{i} \cdot R^{\prime}\left(w_{i}, h L\right)}, \quad R\left(w_{i}, h L\right)=0, \quad,=\frac{d}{d w}, \quad(j=1, \cdots, k)
$$

are called the growth parameters of the modified $k$-step method $(R, S)$.

It follows from (1.6) that

$$
\tilde{\lambda}_{1}=\lambda_{1}=1
$$

Furthermore, we have $\tilde{\lambda}_{j} \neq 0$, and, from (1.7), (1.8), and (1.6),

$$
\tilde{\lambda}_{i}=\lambda_{i}+O(h), \quad 0 \leqq h L<\Omega, j=2, \cdots, k .
$$

Definition. Let the zeros of $R(w, h L)$ be denoted by $w_{i}(j=1, \cdots, k)$. Then define:

$$
\begin{aligned}
& R^{(i)}(w, h L) \equiv R\left(w w_{i}, h L\right) \equiv \sum_{\nu=0}^{k} \tilde{\alpha}_{\nu}^{(j)} \cdot w^{\nu} \\
& S^{(j)}(w, h L) \equiv \tilde{\lambda}_{j}^{-1} \cdot S\left(w w_{i}, h L\right) \equiv \tilde{\lambda}_{i}^{-1} \cdot \sum_{\nu=0}^{k} \tilde{\beta}_{\nu}^{(i)} \cdot w^{\nu},
\end{aligned}
$$

with

$$
\tilde{\alpha}_{\nu}^{(i)}=\tilde{\alpha}_{\nu} \cdot w_{j}^{\nu} \text { and } \tilde{\beta}_{\nu}^{(j)}=\tilde{\beta}_{\nu} \cdot w_{i}^{\nu} \quad(j=1, \cdots, k ; \nu=0, \cdots, k) .
$$

LEMMA 4. The $k$-step methods defined by the polynomials $R^{(i)}(w, h L)$ and $S^{(i)}(w, h L)$ are consistent, i.e.,

$$
R^{(j)}(1, h L)=0
$$

and

$$
\left.\frac{d R^{(j)}(w, h L)}{d w}\right|_{x=1}=S^{(j)}(1, h L) \quad(j=1, \cdots, k) .
$$

For $j=1$, we have $w_{1}=1$ and thus the statement of Lemma 1 .

The proof is similar to that of Lemma 1 and will again be omitted.

Definition. Let the values $s_{0}, s_{1}, \cdots, s_{k-1}$ be functions of $h$, defined for all values of $h$ sufficiently close to zero, such that

$$
\lim _{h \rightarrow 0} s_{m}(h)=y(a)=s \quad(m=0, \cdots, k-1) .
$$


Let $\left\{y_{n}\right\}$ be the solution of the difference equation

$$
\sum_{\nu=0}^{k} \bar{\alpha}_{\nu} \cdot y_{n-\nu}=h \sum_{\nu=0}^{k} \tilde{\beta}_{\nu} \cdot f_{n+\nu}, \quad n=0,1, \cdots,
$$

defined by the initial values $y_{m}=s_{m}(m=0, \cdots, k-1)$. Then the $k$-step method $(R, S)$ is said to be convergent if

$$
\operatorname{Max}_{x_{n} \in[a, b]}\left|y_{n}-y\left(x_{n}\right)\right| \rightarrow 0 \quad \text { as } \quad h \rightarrow 0 \quad(L \text { fixed }) .
$$

Here, $y(x)$ denotes the exact solution of (1.2).

THEOREM 1. The $k$-step methods $\left(R^{(j)}, S^{(j)}\right)(j=1, \cdots, k)$ defined by (2.6) and $(2.7)$ are convergent. For $j=1$, we get as a special case the convergence of the $k$-step method $(R, S)$.

Proof. The proof is essentially the same as the one for the classical result which says that a stable and consistent $k$-step method is convergent (see [5, Theorem 5.10]), except for one important detail: for $h L>0$ and $j=2, \cdots, k$, the $k$-step methods $\left(R^{(j)}, S^{(i)}\right)$ are no longer strongly stable (in the sense defined above). Since the zeros of $R^{(j)}(w, h L)$ are given by

$$
V_{m}^{(i)}=w_{m} \cdot \boldsymbol{w}_{i}^{-1} \quad(m=1, \cdots, k),
$$

we have $\left|V_{m}^{(i)}\right|>1$ for some values of $m$. But, from [1, p. 440],

$$
w_{i}=z_{i}\left(1+\frac{h L}{2} \cdot \frac{1-z_{i}}{z_{j}}+O\left(h^{2}\right)\right) \quad(j=1, \cdots, k),
$$

where $z_{i}=\exp \left(i \cdot \varphi_{i}\right)$, we find for $n \rightarrow \infty, n h=x_{n}-a \leqq b-a, L$ fixed:

$$
\left|\boldsymbol{w}_{m} \cdot \boldsymbol{w}_{j}^{-1}\right|^{n}=\exp \left(\frac{L}{2}\left(x_{n}-a\right) \cdot\left(\cos \varphi_{m}-\cos \varphi_{i}\right)\right)+O(h) .
$$

With these results the proof of Theorem 1 is easily carried out along the lines of the classical proof mentioned above.

3. Elimination of Marginal Stability. In (1.2), let $f_{y y}(x, y(x)) \in C[a, b]$, and assume that the exact solution $y(x)$ be of class $C^{k+4}[a, b]$ (where $k$ denotes the degree of the polynomial $R(w, h L)$ ). Without loss of generality let $a=0$. The difference operator $\tilde{\mathscr{L}}$ associated with the $k$-step method $(R, S)$ is given by

$$
\tilde{L}[y(x) ; h L] \equiv \sum_{\nu=0}^{k}\left(\tilde{\alpha}_{\nu} y(x+\nu h)-h \tilde{\beta}_{\nu} y^{\prime}(x+\nu h)\right) .
$$

Let

$$
\tilde{\mathfrak{L}}=\mathfrak{L}+\frac{h L}{2} \cdot \mathfrak{L}^{*},
$$

where, for all $y(x) \in C^{k+4}[a, b]$ :

$\mathcal{L}[y(x) ; h] \equiv \sum_{\nu=0}^{k}\left(\alpha_{\nu} y(x+\nu h)-h \beta_{\nu} y^{\prime}(x+\nu h)\right)=C_{k+3} \cdot y^{(k+3)}(x) \cdot h^{k+3}+O\left(h^{k+4}\right)$, 
and

$\mathfrak{L}^{*}[y(x) ; h] \equiv \sum_{\nu=0}^{k}\left(p_{\nu} y(x+\nu h)-h q_{\nu} y^{\prime}(x+\nu h)\right)=C_{k+2}^{*} \cdot y^{(k+2)}(x) \cdot h^{k+2}+O\left(h^{k+3}\right)$,

since $\mathscr{L}$ has by hypothesis maximum order $p=k+2$, whereas $\mathfrak{L}^{*}$ has order $p^{*} \geqq$ $k+1$ (see [5, p. 226], [1, p. 442]). Hence,

$$
\tilde{\mathfrak{L}}[y(x) ; h L]=-H(x, L) \cdot h^{k+3}+O\left(h^{k+4}\right),
$$

where

$$
-H(x, L)=C_{k+3} \cdot y^{(k+3)}(x)+\frac{L}{2} C_{k+2}^{*} y^{(k+2)}(x) .
$$

The constants $C_{k+3}$ and $C_{k+2}^{*}$ depend on the coefficients of $\rho(w), \sigma(w)$, and $\rho^{*}(w)$, $\sigma^{*}(w)$, respectively, but are independent of $h L$ and $y(x)$ [5, p. 221], [1, p. 442].

Subtract, now, the expression for $\tilde{L}[y(x) ; h L]$ from the difference equation (2.8) to get

$$
\sum_{\nu=0}^{k} \tilde{\alpha}_{\nu} \cdot e_{n+\nu}-h \sum_{\nu=0}^{k} \tilde{\beta}_{\nu} \cdot g_{n+\nu} \cdot e_{n+\nu}=H\left(x_{n}, L\right) h^{k+3}+h \cdot O\left(e_{n+\nu}^{2}\right)+O\left(h^{k+4}\right),
$$

where $e_{m}=y_{m}-y\left(x_{m}\right)$ denotes the truncation error at the point $x=x_{m}$, and where $g(x)=f_{\nu}(x, y(x)), g_{m}=g\left(x_{m}\right)$.

Suppose that the initial values for $\left\{e_{n}\right\}$ are of the form

$$
e_{j} \equiv \delta_{j}(h)=O\left(h^{a}\right), \quad q \geqq 1, \quad j=0, \cdots, k-1 .
$$

It then follows [6, Lemma 3.2] that the values $e_{n}$ satisfy the relation

$$
e_{n}=O\left(h^{r}\right), \quad r=\min (k+2, q), \quad x_{n} \in[0, b] .
$$

Hence, (3.1) becomes

$$
\sum_{\nu=0}^{k} \tilde{\alpha}_{\nu} e_{n+\nu}-h \sum_{\nu=0}^{k} \tilde{\beta}_{\nu} g_{n+\nu} \cdot e_{n+\nu}=H\left(x_{n}, L\right) h^{k+3}+O\left(h^{k+4}\right)+O\left(h^{2++1}\right) .
$$

The solution of (3.3) can be written as the sum of the solution of the homogeneous equation

$$
\sum_{\nu=0}^{k}\left(\tilde{\alpha}_{\nu}-h \tilde{\beta}_{\nu} g_{n+\nu}\right) \cdot e_{n+\nu}=0
$$

with initial values (3.2), and the solution of the inhomogeneous equation (3.3) with initial values being equal to zero. It can be shown [5, p. 250] that the solution of the inhomogeneous difference equation does not depend on the growth parameters $\bar{\lambda}_{i}$ of the $k$-step method $(R, S)$, and it, therefore, has no influence on the stability of the general solution $\left\{e_{n}\right\}$ of (3.3). For this reason, we shall only consider the homogeneous equation (3.4). Its solution shall be represented in the form

$$
e_{n}=\sum_{i=1}^{k} A_{i} \cdot e_{n}^{(i)},
$$


where the sequences $\left\{e_{n}^{(i)}\right\}, j=1, \cdots, k$, form a fundamental system of solutions of (3.4) at the point $n=0$. In order to construct such a fundamental system, consider the differential equation $z^{\prime}(x)=g_{0} \cdot z(x)$. If we apply the method $(R, S)$ to solve this equation, we get a linear difference equation with constant coefficients, namely,

$$
\sum_{\nu=0}^{k}\left(\tilde{\alpha}_{\nu}-h g_{0} \tilde{\beta}_{\nu}\right) z_{n+\nu}=0 .
$$

Let $v_{j}(j=1, \cdots, k)$ be the solutions of the equation

$$
R(w, h L)-h g_{0} \cdot S(w, h L)=0 .
$$

According to Lemma 2, these roots have multiplicity one whenever $h$ is sufficiently close to zero, and, thus, the determinant

$$
W_{0}=\left|\begin{array}{cccc}
1 & v_{1} & \cdots & v_{1}^{k-1} \\
\vdots & & \\
1 & v_{k} & \cdots & v_{k}^{k-1}
\end{array}\right|
$$

is different from zero for such values of $h$. If we define the sequences $\left\{e_{n}^{(i)}\right\}$ by setting

$$
e_{m}^{(j)}=v_{i}^{m}, \quad m=0, \cdots, k-1, j=1, \cdots, k,
$$

then these sequences form a fundamental system of solutions of (3.4) at $n=0$, and we may represent the solution of (3.4) in the form (3.5) (see also [5, p. 211]).

In order to establish asymptotic relations for the expressions $e_{n}^{(i)}$, let

$$
\xi_{n}^{(i)} \equiv e_{n}^{(i)} \cdot w_{i}^{-n},
$$

where $R\left(w_{i}, h L\right)=0, j=1, \cdots, k$. For $m=0, \cdots, k-1$, we have

$$
\xi_{m}^{(i)}=1+O(h)
$$

It follows from the definition of the sequences $\left\{e_{n}^{(i)}\right\}$ that the sequences $\left\{\xi_{n}^{(i)}\right\}$ satisfy (3.4), i.e.,

$$
\sum_{\nu=0}^{k}\left(\tilde{\alpha}_{\nu}^{(i)}-h \tilde{\lambda}_{j} g_{n+\nu} \tilde{\beta}_{\nu}^{(i)}\right) \cdot \xi_{n+\nu}^{(i)}=0,
$$

since $w_{i} \neq 0$ near $h=0$. The coefficients $\tilde{\alpha}_{v}^{(j)}, \widetilde{\beta}_{v}^{(j)}$ are defined by (2.6) and (2.7).

By Lemma 4 the $k$-step methods $\left(R^{(i)}, S^{(i)}\right)$ are consistent, and by Theorem 1 they are convergent. If we now solve the initial-value problem

$$
\frac{d \xi^{(j)}(x)}{d x}=\tilde{\lambda}_{i} \cdot g(x) \cdot \xi^{(i)}(x), \quad \xi^{(i)}(0)=1,
$$

by the method $\left(R^{(i)}, S^{(i)}\right)$, there results again difference equation (3.7). It follows [5, p. 248] that

$$
\xi_{n}^{(i)}=\xi^{(i)}\left(x_{n}\right)+O(h), \quad j=1, \cdots, k, \quad x_{n} \in[0, b] .
$$

Hence, we find from (3.6)

$$
e_{n}^{(i)}=w_{i}^{n} \cdot \xi^{(i)}\left(x_{n}\right)+O(h), \quad j=1, \cdots, k .
$$


By (2.9), we get for $n \rightarrow \infty, n h=x_{n}, L$ fixed, using the explicit expressions for the functions $\xi^{(i)}(x)$,

$e_{n}^{(i)}=\exp \left(i n \varphi_{i}\right) \cdot \exp \left(\frac{L}{2} \cdot \frac{1-z_{j}}{z_{i}} x_{n}+\tilde{\lambda}_{j} \cdot \int_{0}^{x_{n}} g(x) d x\right)+O(h), \quad(j=1, \cdots, k)$.

With these relations, (3.5) takes the final form

$e_{n}=\sum_{j=1}^{k} A_{i} \cdot \exp \left(i n \varphi_{i}\right) \cdot \exp \left(\frac{L}{2} \cdot \frac{1-z_{i}}{z_{i}} x_{n}+\lambda_{i} \cdot \int_{0}^{x_{n}} g(x) d x\right)+O(h), \quad x_{n} \in[0, b]$.

Here we have used (2.5). Let

$$
r_{i}(x) \equiv \exp \left(i n \varphi_{i}\right) \cdot \exp \left(\frac{L}{2} \cdot \frac{1-z_{i}}{z_{i}} x+\lambda_{i} \cdot \int_{0}^{x} g(t) d t\right) \quad(j=1, \cdots, k) .
$$

Following the terminology of Hull and Newbery [8], we introduce the notions of relative and absolute stability:

Definition. The $k$-step method $(R, S)$ is called relatively stable (in the asymptotic sense: $h \rightarrow 0, n h=x_{n}, L$ fixed) with respect to the initial-value problem (1.2) if for all $x_{n} \in[0, b]$ and all $j=2, \cdots, k$ :

$$
\left|r_{i}\left(x_{n}\right)\right|<\left|r_{1}\left(x_{n}\right)\right| .
$$

Definition. The $k$-step method $(R, S)$ is called absolutely stable (in the asymptotic sense stated above) with respect to the initial-value problem (1.2) if for all $x_{n} \in[0, b]$ and all $j=2, \cdots, k$ :

$$
\left|r_{i}\left(x_{n}\right)\right|<1 .
$$

From the fact that the growth parameters $\lambda_{i}$ of the $k$-step method $(\rho, \sigma)$ are real [4, p. 40], we obtain, without difficulty, the following results:

THEOREM 2. The $k$-step method $(R, S)$ is relatively stable (in the asymptotic sense) with respect to the initial-value problem (1.2) if the parameter $L$ satisfies the inequality

$$
L>2 \cdot \operatorname{Max}_{i=2, \cdots, k ; x_{n} \in[0, b]} \frac{\left(\lambda_{i}-1\right) \cdot \int_{0}^{x_{n}} g(x) d x}{\left(1-\cos \varphi_{j}\right) \cdot x_{n}} .
$$

THEOREM 3. The $k$-step method $(R, S)$ is absolutely stable (in the asymptotic sense) with respect to the initial-value problem (1.2) if the parameter $L$ satisfies the inequality

$$
L>2 \cdot \operatorname{Max}_{i=2, \ldots, k ; x_{n} \in[0, b]} \frac{\lambda_{i} \cdot \int_{0}^{x_{n}} g(x) d x}{\left(1-\cos \varphi_{i}\right) \cdot x_{n}} .
$$

4. Optimal $k$-Step Methods of Open Type. Let $\rho(z)$ be the first characteristic polynomial of an optimal $k$-step method as given by (1.3). If we set

$$
\sigma_{0}(z)=\sum_{\nu=0}^{k-1} c_{\nu}(z-1)^{\nu}
$$


where

$$
\rho(z) \cdot(\log z)^{-1}=\sum_{\nu=0}^{\infty} c_{\nu}(z-1)^{\nu} \quad(\log 1=0)
$$

then the resulting $k$-step method is a method of open type and is known to have order $p=k[3, \mathrm{p} .52]$. Such a method has always a number of negative growth parameters [4, p. 42] and is therefore subject to marginal stability.

It is obvious that the arguments presented in Section 2 and Section 3 are easily modified for the case of an optimal $k$-step method of open type as described above. Except for the values of the growth parameters $\lambda_{j}$, the two theorems stated at the end of Section 3 remain valid if $(R, S)$ is now an open method given by (1.7) and

$$
S(w, h L)=\sigma_{0}(w)+\frac{h L}{2} \sigma_{0}^{*}(w)
$$

where

$$
\sigma_{0}^{*}(w)=\sum_{\nu=0}^{k-1} \gamma_{\nu}(w-1)^{v}
$$

(see (1.10) and (1.11)).

In the following section a particular $k$-step method of open type, the modified midpoint rule, will be used to solve a nonlinear differential equation. Other methods (for $k=4$ and $k=6$ ) are contained in [2, pp. 67-75].

5. Numerical Illustrations.

I. Consider the simple initial-value problem

$$
y^{\prime}=10 x-10 x y, \quad y(0)=0, \quad 0 \leqq x \leqq 3 .
$$

Its exact solution is $y(x)=1-\exp \left(-5 x^{2}\right)$. For the numerical integration of (4.1) we use the method of Milne-Simpson and its modification [1]:

$$
\begin{array}{r}
y_{n+2} \cdot(1+h L)-h L \cdot y_{n+1}-y_{n} \\
=\frac{h}{12} \cdot\left[(4+5 h L) \cdot f_{n+2}+(16+8 h L) \cdot f_{n+1}+(4-h L) \cdot f_{n}\right] \\
\quad(n=0,1, \cdots) .
\end{array}
$$

The growth parameters of the classical method $(L=0)$ are

$$
\lambda_{1}=1, \quad \lambda_{2}=-\frac{1}{3},
$$

and the inequality for absolute stability becomes (for the interval indicated above) $L>5$. In order to have also relative stability the parameter $L$ must satisfy $L>20$.

The following table contains some numerical values corresponding to the stepsize $h=0.02$. The two initial values for $y_{n}$ were exact, i.e., $y_{0}=1$ and $y_{1}=1-\exp \left(-5 h^{2}\right)$. 
All computations were performed on the IBM $360 / 50$ of the Dalhousie University Computer Centre.

\begin{tabular}{c|r|r|c}
\hline$x_{n}$ & $\begin{array}{r}L=0 \\
10^{9} \cdot e_{n}\end{array}$ & $\begin{array}{c}L=10 \\
10^{9} \cdot e_{n}\end{array}$ & $\begin{array}{c}L=20 \\
10^{9} \cdot e_{n}\end{array}$ \\
\hline$\vdots$ & & & \\
1.20 & & -38 & -73 \\
1.22 & -21 & -33 & -65 \\
1.24 & 33 & -29 & -57 \\
1.26 & -26 & -25 & -50 \\
1.28 & 38 & -22 & -43 \\
1.30 & -33 & -18 & -37 \\
$\vdots$ & & & \\
1.50 & -91 & -2 & -6 \\
1.52 & 103 & -2 & -4 \\
1.54 & -112 & -2 & -4 \\
1.56 & 125 & -1 & -3 \\
1.58 & -138 & -1 & -2 \\
1.60 & 154 & -1 & -2 \\
$\vdots$ & & & \\
2.90 & -2212811 & 0 & 0 \\
2.92 & 2672923 & 0 & 0 \\
2.94 & -3232706 & 0 & 0 \\
$\vdots$ & & & \\
\hline
\end{tabular}

II. The nonlinear problem

$$
y^{\prime}=-2 x y^{2}, \quad y(0)=1, \quad 0 \leqq x \leqq 10,
$$

whose exact solution is

$$
y(x)=1 /\left(1+x^{2}\right)
$$

was solved numerically by the midpoint rule and its modification [2]:

$y_{n+2}(1+h L)-h L \cdot y_{n+1}-y_{n}=\frac{h}{2} \cdot\left[(4+3 h L) \cdot f_{n+1}-h L \cdot f_{n}\right] \quad(n=0,1, \cdots)$.

The growth parameters of the classical method are

$$
\lambda_{1}=1, \quad \lambda_{2}=-1,
$$

and a simple calculation yields the inequalities $L>1.609$ for absolute stability, and $L>3.219$ for relative stability (for the interval $0 \leqq x \leqq 10$ ). The initial values for $\left\{y_{n}\right\}$ were $y_{0}=1$ (exact initial value), $y_{1}$ exact to eight places after the decimal point. 


\begin{tabular}{|c|c|c|c|c|}
\hline$x_{n}$ & $y\left(x_{n}\right)$ & $\begin{array}{c}L=0 \\
e_{n}\end{array}$ & $\begin{array}{c}L=1.5 \\
e_{n}\end{array}$ & $\begin{array}{c}L=3.0 \\
e_{n}\end{array}$ \\
\hline \multicolumn{5}{|l|}{ : } \\
\hline 1.0 & 0.5000000 & -0.0019152 & -0.0017987 & -0.0018697 \\
\hline 1.1 & 0.4524887 & -0.0007246 & -0.0012294 & -0.0014397 \\
\hline 1.2 & 0.4098361 & -0.0015512 & -0.0011978 & -0.0011960 \\
\hline 1.3 & 0.3717472 & 0.0000025 & -0.0006647 & -0.0008499 \\
\hline \multicolumn{5}{|l|}{$:$} \\
\hline 5.0 & 0.0384615 & -0.0741068 & -0.0000639 & 0.0000471 \\
\hline 5.1 & 0.0370233 & 0.0438333 & 0.0001428 & 0.0000455 \\
\hline 5.2 & 0.0356633 & -0.0846457 & -0.0000562 & 0.0000427 \\
\hline 5.3 & 0.0343761 & 0.0414900 & 0.0001274 & 0.0000411 \\
\hline \multicolumn{5}{|l|}{ : } \\
\hline 7.5 & 0.0174672 & -0.1884251 & 0.0000318 & 0.0000153 \\
\hline 7.6 & 0.0170184 & -0.2491781 & -0.0000029 & 0.0000147 \\
\hline 7.7 & 0.0165865 & -0.3513947 & 0.0000280 & 0.0000141 \\
\hline 7.8 & 0.0161708 & -0.5935879 & -0.0000014 & 0.0000137 \\
\hline \multicolumn{5}{|l|}{ : } \\
\hline 9.7 & 0.0105163 & $\ldots$ & 0.0000087 & 0.0000068 \\
\hline 9.8 & 0.0103050 & $\ldots$ & 0.0000038 & 0.0000066 \\
\hline 9.9 & 0.0100000 & $\ldots$ & 0.0000079 & 0.0000064 \\
\hline 10.0 & 0.0099010 & $\ldots$ & 0.0000039 & 0.0000062 \\
\hline
\end{tabular}

Other modified methods (for $k=4$ and $k=6$; [1], [2]) show a very similar error behavior.

Acknowledgement. I would like to thank Professor P. Henrici for his interest in my work and for his many helpful suggestions. I also wish to thank Mrs. Patricia Bewers (Dalhousie University Computer Centre) for her help in programming the results.

Department of Mathematics

Dalhousie University

Halifax, Nova Scotia, Canada

1. H. Brunner, "Stabilization of optimal difference operators," Z. Angew. Math. Phys., v. 18, 1967, pp. 438-444. MR 36 \#1111.

2. H. BRUNNER, Stabilisierung optimaler Differenzenverfahren zur numerischen Integration gewöhnlicher Differentialgleichungen, Dissertation, Juris Verlag, Zürich, 1969.

3. G. DAHLQuIST, "Convergence and stability in the numerical integration of ordinary differential equations," Math. Scand., v. 4, 1956, pp. 33-53. MR 18, 338.

4. G. DAHLQUIST, "Stability and error bounds in the numerical integration of ordinary differential equations," Kungl. Tekn. Högsk. Handl. Stockholm, No. 130, 1959. MR 21 \#1706. 
5. P. HENRICI, Discrete Variable Methods in Ordinary Differential Equations, Wiley, New York, 1962. MR 24 \#B1772.

6. P. HenRICI, Error Propagation for Difference Methods, Wiley, New York, 1963. MR $27 \# 4365$.

7. M. MARden, Geometry of Polynomials, 2nd. ed., Math. Surveys, no. 3, Amer. Math. Soc., Providence, R.I., 1966. MR 37 \#1562.

8. T. E. HULl \& A. C. R. NeWBERY, "Corrector formulas for multi-step integration methods," J. Soc. Indust. Appl. Math., v. 10, 1962, pp. 351-369. MR 27 \#2130. 